\title{
Utilisation d'une montmorillonite, la terre de Bezenet, dans le dosage sélectif de la créatinine sanguine des ruminants domestiques
}

\author{
por' CI. LABOUCHE
}

Au cours d'une étude de la filtration glomérulaire rénale chez des ruminants domestiques, nous avons dosé la créatinine du sérum sanguin selon JAFFE. Par cette méthode, une molécule de créatinine sanguine s'unit à une molécule d'acide picrique ainsi qu'à un nombre variable de molécules de soude et d'eau pour donner des composés de couleur rouge.

La réaction n'est pas spécifique. L'acétone, l'acide acétylacétique, le méthylglyoxal, l'acide glycuronique, l'acide ascorbique, certains glucides et divers protides peuvent la foumir. Des techniques de destruction de ces chromogènes ont été proposées, mais le plus souvent on préfère extraire la créatinine en l'adsorbant sur une substance appropriée (réactif de LLOYD, bentonites commerciales) (1) (2). La créatinine éluée subit alorș la coloration, à l'abri des interventions parasites.

Les agents adsorbants classiques nous faisant défaut, nous avons pensé qu'une argile montmorillonite, la terre de BEZENET (Allier) ou Jagolithe, dont MEUNIER avait autrefois proposé l'utilisation pour classer les huiles de poisson suivant leur richesse en vitomine A (3) pourrait peut-être les remplacer.

Cette terre, de couleur grise, est formée de grains de taille variable ainsi que le montre l'essai de tamisage résumé ci-dessous.

Rev, Elév. Méd, vét. Pays trop., 1963, no 1.

Reçu pour publication : juillet 1962.

$\begin{array}{ccc}\begin{array}{c}\text { Traversant le } \\ \text { tamis }\end{array} & \begin{array}{c}\text { Arrêté par } \\ \text { le tamis } \\ \text { No }\end{array} & \begin{array}{c}\text { P. } 100 \text { du poids } \\ \text { total de l'argile } \\ \text { tamisée }\end{array} \\ - & - & - \\ 45 & 45 & 0,06 \\ 60 & 60 & 2,7 \\ 80 & 80 & 46,8 \\ 100 & 100 & 30,4 \\ 190 & 190 & 16,9 \\ & - & 3,2\end{array}$

La Jagolithe est colorée en bleu par la benzidine en solution acétique, en vert clair par l'aniline et en brun rouge par la paraphénylènediamine. La réaction est acide en présence d'une solution aqueuse d'hélianthine.

Avant de l'utiliser pour le dosage de la créatinine, nous avons envisagé les points suivants :

- pouvoir adsorbant et infivence de la granulation

- action sur les spectres d'absorption lumineuse

- action sur la couleur des solutions alcalines d'acide picrique

- vérification de la loi de BEER-LAMBERT

- essais de récupération des surcharges de créatinine

- sélectivité de l'adsorption

$$
\begin{aligned}
& \text { 1. - Etude du pouvoir adsorbant } \\
& \text { de la Jagolithe }
\end{aligned}
$$

On évalue le pouvoir adsorbant de l'argile, en mesurant la plus petite quantité capable de 
fixer un poids donné de créatinine en présence d'acide sulfurique et de tungstate de sodium, agents déféquants habituellement préconisés dans le dosage de la créatinine sanguine. (1)

Dans des tubes à centrifuger coniques, on place successivement :

Solution de créctinine ( 6 ou $\left.10 \gamma / \mathrm{cm}^{3}\right) . .2 \mathrm{ml}$ Eau distillée .................. $3 \mathrm{ml}$

Na tungstate (solution à 10 p. 100) ... $1 \mathrm{ml}$ $\mathrm{H}_{2} \mathrm{SO}_{4}(0,67 \mathrm{~N}) \ldots \ldots \ldots \ldots \ldots \ldots 2 \mathrm{ml}$

Après homogénéisation du mélange, on ajoute des quantités variables de terre de Bezenet. On agite trois fois en dix minutes à l'aide d'une baguette de verre, puis on centrifuge à grande vitesse pendant dix minutes. Le surnageant est enlevé et les tubes sont retournés sur du papier filtre afin de réaliser un égouttage aussi parfait que possible du culot. Pendant ce temps on prépare un tube témoin contenant seulement deux centimètres cubes de la solution de créatinine et un «blanc» constitué par deux centimètres cubes d'eau distillée.

On ajoute ensuite à tous les tubes $7.5 \mathrm{~cm}^{3}$ de réactif picro-sodé ainsi que $2 \mathrm{~cm}^{3} \mathrm{~d}^{\text {eau distillée }}$ à ceux contenant de l'argile afin de rétablir l'égalité de volume avec le tube témoin ef le blanc. Après agitation et dix minutes de contact, on centrifuge pendant dix minutes. La densité optique des surnageants est mesurée au spectrophotomètre Beckman à $520 \mathrm{~m} \mu$ et exprimée en pourcentage de la densité optique du témoin.

a) Pouvor adsorbont de la Jagolithe non tamisée.

La solution de créatinine utilisée contenait $6 \gamma / \mathrm{cm}^{3}$

\begin{tabular}{cc}
$\begin{array}{c}\text { Quantité } \\
\text { d'argile }\end{array}$ & $\begin{array}{c}\text { Densité optique } \\
\text { du surnageant } \\
\text { (p) } 100 \text { de la densité } \\
\text { optique du témoin) }\end{array}$ \\
\hline 0 & $-\overline{100}$ \\
0,1 & 75 \\
0,2 & 111 \\
0,4 & 109
\end{tabular}

L'adsorption est complète à partir de 0,2 g. L'actıvité est donc inférieure à celle du réactif de Lloyd ou d̀ celle des bentonites pour lesquels la quantité maximale fixée est de 1 p.'100 du poids de l'adsorbant. On remarquera également que lorsque l'adsorption est complète, la densité optique est supérieure à celle du témoin d'environ 10 p. 100.

b) Pouvoir adsorbant ef granulation de lo jagolithe.

Nous avons vérifié l'influence de la grosseur des grains d'argile sur le pouvoir adsorbant par un protocole identique au précédent mais en utilisant des solutions à $10 \mu \mathrm{g}$ de créatinine au ml. L'argile est passée à travers une série de tamıs en bronze de numérotation : $60,80,100$ et 190 . On a ainsi obtenu les fractions suivantes:

A : traverse la maille 190

B. : traverse la maille 100 et est retenue par la maille 190

C: traverse la maille 80 et est retenue par la maille 100

$D$ : traverse la maille 60 ef est retenue par la maille 80

$E$ : est retenue par la maille 60

Les pourcentages moyens de créatinine fixée en fonction du poids de l'argile et de la grosseur du grain de Jagolithe sont réunis dans le tableau suivant.

\begin{tabular}{|c|c|c|c|c|c|c|c|}
\hline \multirow{2}{*}{ Fraction } & \multicolumn{7}{|c|}{ Pofda d'artile on oent1gramges. } \\
\cline { 2 - 8 } & 5 & 10 & 20 & 40 & 60 & 80 & 100 \\
\cline { 2 - 8 } A & 86 & 95 & $101^{\prime}$ & 56 & & & \\
B & 54 & 74 & 96 & 103 & 105 & & \\
C & 34 & 50 & 71 & 97,5 & 108 & & \\
D & & 40 & 51 & 80 & 85 & 85 & 60 \\
E & & & 55 & 85 & 81 & 86 & 74 \\
\hline
\end{tabular}

On remarquera :

$1^{\circ}$ Que la quantité d'argile nécessaire pour adsorber $20 \mu \mathrm{g}$ de créatınıne est de $20 \mathrm{cg}$ pour $\mathrm{A}$, de 20 à $40 \mathrm{cg}$ pour $B$, de $40 \mathrm{~d} 60 \mathrm{cg}$ pour $C$.

$2^{\circ}$ Que les fractions les plus grossières ( $D$ et $E$ ) ne semblent pas permettre une fixation complète.

$3^{0}$ Qu'un excès d'argile se traduit par une diminution du pourcentage de rétention $(A, D, E$,$) .$

$4^{\circ}$ Que lorsque la récupération de la créatinine est complète, elle est légèrement supérieure à 100 p 100. 
Nous avons alors essayé de confirmer $\left(2^{\circ}\right)$ et (30) de la manière suivante : Le surnageant de la première centrifugation est recueilli et on $y$ ajoute $20 \mathrm{cg}$ de la fraction $\mathrm{A}$, quantité que nous venons de vérifier comme suffisante pour adsorber la créatinine qui n'aurait pas été retenue. On opère ensuite comme précédemment. En aucun cas, nous n'avons pu mettre en évidence la présence de créatinine dans les surnageants lorsqu'on utilise $40 \mathrm{cg}$ de $A_{1} 60,80,100 \mathrm{cg}$ de $D$ ou de $\mathrm{E}$.

L'adsorption est donc dans tous les cas complète, mais les capacités d'absorption lumineuse ont été sans doute modifiées.

\section{2. - Influence de la Jagolithe sur les spectres d'absorption des complexes créatinine-acide picrique.}

La coloration a été développée à partir d'une solution pure de créatinine sans utilisation de l'argile (coloration témoin) et après action de quantités variables de Jagolithe (fractions $A$, D. E,)

La densité optique est déterminée pour des lumières de longueur d'onde comprise entre 450 et $560 \mathrm{~m} \mu$. Pour chaque spectre, on égale la densité optique maximale à 100 , les cutres D. 0 . étant alors exprimées en pour cent du maximum d'absorption.

Les résultats obtenus sont consignés dans le tableau figuré à la page suivante:

Les spectres correspondants sont représentés dons les figures 1,2 , et 3 .

On remarquera que le maximum d'absorption lumineuse situé à $480 \mathrm{~m} \mu$ pour le témoin se déplace vers les grandes longueurs d'onde en présence de l'argile. On note ainsi, suivant les cas, des maxima d'absorption à 495, 500, 505 . $510 \mathrm{~m} \mu$ tandis que l'absorption vers' les courtes longueurs d'onde diminue.

Ces déformations spectrales permettent d'expliquer que les récupérations de créatinine puissent être supérieures à 100 p. 100.

En conséquence nous pouvons déjc̀ dire que lors d'un dosage éventuel de la créatinine au moyen de Jagolithe, les solutions étalons devront être traitées par les mêmes quantités d'argile de même qualité que les échantillons soumis aux dosages.

\section{3. - Influence de la Jagolithe sur la coloration de ta solution alcaline d'acide picrique}

Au cours de l'élution de la créatinine et du développement de la coloration, un excédent de réactif picro-sodé reste inemployé. On peut se demander, par conséquent, si sa couleur propre ne pourrait pas être modifiée par contact avec l'argile.

Nous avons done comparé l'absorption lumineuse du réactif avant et après contact avec la Jagolithe $\left(20 \mathrm{cg}\right.$ de fraction A pour $7,5 \mathrm{~cm}^{3}$ de réactif) le réactif étant séparé ensuite de l'argile. par centrifugation. On note alors une légère intensification. de la couleur, la solution ne laissant alors passer que 98 p. 100 de la lumière traversant le réactif seul.

Il sera donc nécessaire de traiter les blancs de la même manière que les échantitlons à doser.

\section{4. - Vérification de la loi de BEER-LAMBERT avec des solutions pures de créatinine}

La technique 'suivante à été suivie. Des gammes de concentrations croissantes sont ainsi réalisées.

\begin{tabular}{|c|c|c|c|c|}
\hline & tarbe 0 & trube 1 & tube 2 & tube 3 \\
\hline $\begin{array}{l}\text { soiution de créatintre } \\
\text { aे } 10 \mu \mathrm{g} / \mathrm{ml} \text {, ml }\end{array}$ & 0 & 1 & 2 & 3 \\
\hline Esul disłlliée, mfl & 3 & 2 & 1 & 0 \\
\hline lia tungstate $10 \mathrm{p}, 100, \mathrm{ml}$ & 1 & 1 & 1 & 1 \\
\hline $\mathrm{SO}_{4} \mathrm{H}_{2},(0,67 \mathrm{~N})$, m] & 2 & 2 & 2 & 2 \\
\hline
\end{tabular}

Suivant les cas, on utilise les quantités suivantes d'argile :

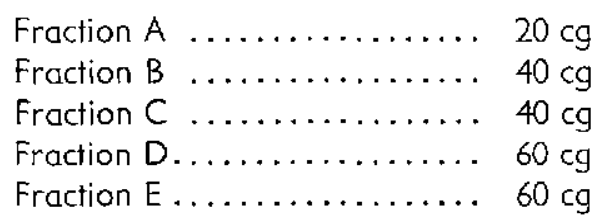

On vérifie que l'adsorption est complète pour les concentrations les plus élevées de créatinine, par la méthode exposée en $(2, b)$ 


\begin{tabular}{|c|c|c|c|c|c|c|c|c|c|c|}
\hline \multirow{2}{*}{${ }_{\mathrm{mpa}}^{\lambda}$} & \multirow{2}{*}{ témoin } & \multicolumn{2}{|c|}{ Fraction A } & \multicolumn{4}{|c|}{ Fraction B } & \multicolumn{3}{|c|}{ Fraction $\mathrm{E}$} \\
\hline & & $20 \mathrm{cg}$ & $40 \mathrm{cg}$ & $40 \mathrm{cg}$ & $60 \mathrm{cog}$ & $80 \mathrm{cg}$ & $100 \mathrm{cE}$ & $60 \mathrm{cg}$ & $80 \mathrm{cg}$ & $100 \mathrm{cE}$ \\
\hline 450 & 60 & 66 & 46 & - & - & - & - & 56 & 51 & 6 \\
\hline 460 & $B 2$ & 94 & 72 & - & 65 & 56 & 11 & 64 & 52 & 2 \\
\hline 465 & 84 & - & - & - & 68 & 58 & 11 & 66 & 56 & - \\
\hline 470 & 93 & 95 & 78 & 63 & 71 & 60 & 10 & 71 & 56 & 4 \\
\hline 475 & 98 & 98 & 81 & 68 & 73 & 60 & 13 & 74 & 58 & - \\
\hline 480 & 100 & 97 & $T 7$ & 74 & 79 & 69 & 27 & 81 & 68 & 21 \\
\hline 485 & 99 & 95 & 69 & 86 & 89 & 83 & 57 & 89 & 82 & 51 \\
\hline 490 & 96,5 & 99 & 87 & 95 & 96 & 93 & 78 & 96 & 93 & 74 \\
\hline 495 & 93.5 & 100 & 90 & 99 & 99 & 99,5 & 91 & 99 & 97 & 88 \\
\hline 500 & 89,5 & 99 & 95 & 100 & 100 & 100 & 98,5 & $\underline{100}$ & 100 & 96 \\
\hline 505 & 84 & 98 & 99 & 97 & 97 & 99 & 100 & 98 & 99 & 100 \\
\hline 510 & 78 & 95 & 100 & 93 & 94 & 95,5 & 98,5 & 94 & 96 & 99 \\
\hline 515 & 71 & 88 & 96 & - & 98 & 90,5 & 93 & 88 & $B 9$ & 94 \\
\hline 520 & 61 & 80 & 90 & 76 & 78 & 80 & 84 & 78 & 80 & 88 \\
\hline 525 & 52 & - & - & - & 68 & 70 & 73 & - & - & - \\
\hline 530 & 43 & 61 & 72 & 56 & 57 & 59 & 61 & 57 & 58 & 66 \\
\hline 535 & 34 & - & - & - & 47 & 49 & 51 & - & - & - \\
\hline 540 & 26 & 40 & 51 & 37 & 37 & 38 & 41 & 38 & 38 & 46 \\
\hline 545 & 19 & - & - & - & 30 & 31 & 33 & - & - & - \\
\hline 550 & 14,5 & 26 & 37 & 24 & 24 & 25 & 26 & 24 & 24 & 32 \\
\hline 560 & 8 & - & - & 15 & 15 & 16 & 17 & 8 & 16 & 22 \\
\hline
\end{tabular}




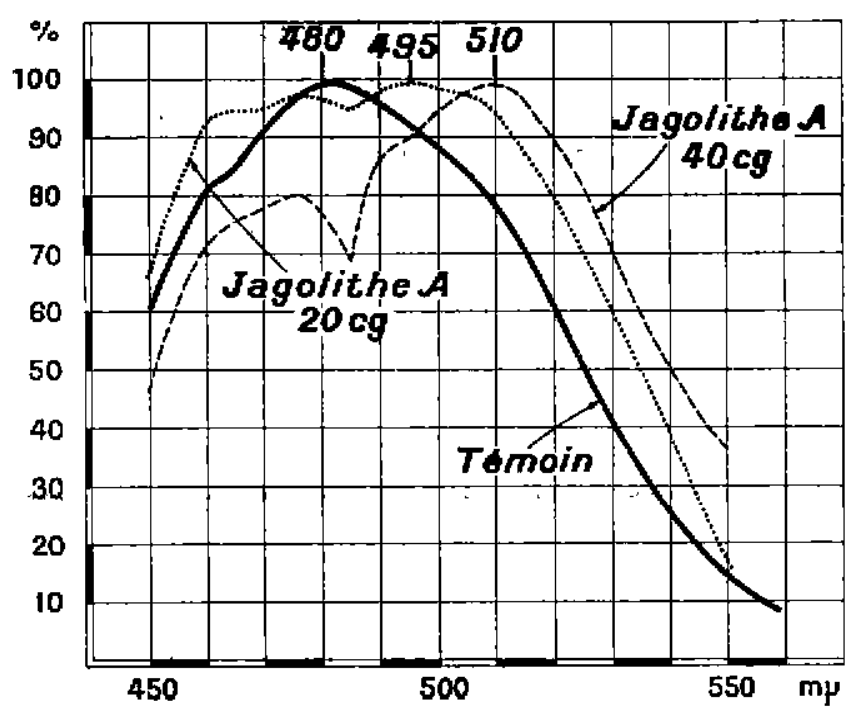

Fig. 1. - Modification du spectre d'absorption lumineuse du complexe acide picrique-créatinine sous I'influence de quantités variables de Jagolithe $A$.

Fig. 2. - Modification du spectre d'absorption lumineuse du complexe acide picrique-créatinine sous l'influence de quantités variables de Jagolithe $E$.

Fig. 3. - Modification du spectre d'absorption lumineuse du complexe acide picrique-créatinine sous l'influence de quantités variables de Jagolithe $E$.

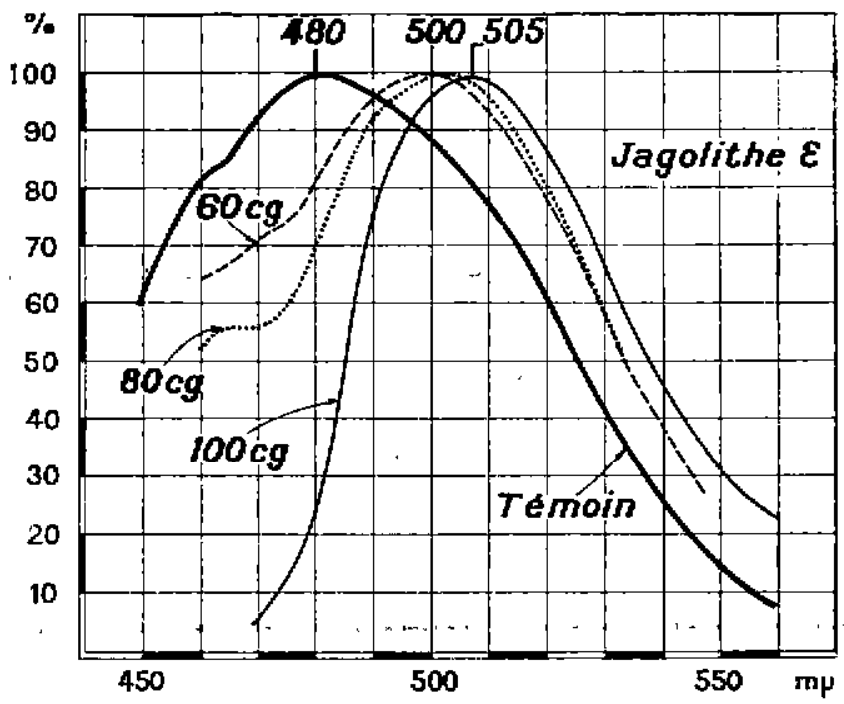


Les densités optiques sont mesurées à différentes longueurs d'onde et la droite de régression concentration $=f$ (densité optique) est calculée par la technique des moindres carrés. Les valeurs calculées sont alors comparées aux concentrations réelles et l'erreur relative est évaluée.

A titre indicatif, nous reproduisons ici les résultats obtenus avec la Jagolithe A $(20 \mathrm{cg})$.
La précision est bonne à toutes les longueurs d'onde et la droite de régression passe très près de l'origine des axes de coordonnées.

Par contre la concordance est moins sátisfaisante entre les valeurs calculées ef les valeurs réelles pour les granulations plus fortes ef l'erreur augmente avec la grosseur des grains, ainsi que le montre le tableau ci-dessous obtenu pour une $\lambda=470 \mathrm{~m} \mu$.

\begin{tabular}{|c|c|c|c|c|c|c|c|}
\hline \multirow{3}{*}{$\begin{array}{l}\lambda \\
\text { max. }\end{array}$} & \multirow{3}{*}{$\begin{array}{l}\text { Equation de régression } \\
\mu g \text { Cráatinine =f. (D.0.) }\end{array}$} & \multicolumn{6}{|c|}{ Quantité de créatinine par tube à essaj, en $\mu \mathrm{g}$} \\
\hline & & \multicolumn{2}{|c|}{10} & \multicolumn{2}{|c|}{20} & \multicolumn{2}{|c|}{30} \\
\hline & & $\begin{array}{c}\text { quantité } \\
\text { calculée } \\
\mu_{E}\end{array}$ & $\begin{array}{c}\text { Brreur } \\
\text { relative } \\
\%\end{array}$ & $\begin{array}{c}\text { quantité } \\
\text { calculée } \\
\mu g\end{array}$ & $\begin{array}{c}\text { Erreur } \\
\text { relative } \\
\%\end{array}$ & $\begin{array}{c}\text { quantité } \\
\text { calculée } \\
\mu_{g}\end{array}$ & $\begin{array}{c}\text { Erreur } \\
\text { relative } \\
\%\end{array}$ \\
\hline 470 & $c x=-0,12+82,7 \quad D, 0$ & 10,05 & 0,5 & 19,97 & 0,15 & 30,02 & 0,06 \\
\hline 480 & $\mathrm{Cr}=-0,09+71,7 \mathrm{D} .0$ & 10,09 & 0,9 & 20,12 & 0,6 & 29,88 & 0,4 \\
\hline 490 & $C_{r}=-0,05+70,5$ D. 0. & 10,03 & 0,3 & 20,11 & 0,5 & 29,91 & 0,3 \\
\hline 500 & $\mathrm{Cr}=-0,10+73,6 \mathrm{D} .0$ & 10,05 & 0,25 & 20,21 & 1 & 29,85 & 0,45 \\
\hline 510 & $C_{T}=-0,02+84,5 \mathrm{D}, 0$ & 9.95 & 0,5 & 20,17 & 0,85 & 29,9 & 0,33 \\
\hline
\end{tabular}

\begin{tabular}{|c|c|c|c|c|c|c|c|}
\hline \multirow[b]{3}{*}{ irraction } & \multirow{3}{*}{$\begin{array}{l}\text { Equation de régression } \\
p E \text { Créatinine }=f .(D .0 .)\end{array}$} & \multicolumn{6}{|c|}{ Quantité de créatinire par tube à essai, en $\mu g$} \\
\hline & & \multicolumn{2}{|c|}{10} & \multicolumn{2}{|c|}{20} & \multicolumn{2}{|c|}{30} \\
\hline & & $\begin{array}{c}\text { quantité } \\
\text { calculée } \\
\mu_{g}\end{array}$ & $\begin{array}{c}\text { Errour } \\
\text { relative } \\
\%\end{array}$ & $\begin{array}{c}\text { quantité } \\
\text { calculée } \\
\mu \mathrm{g}\end{array}$ & $\begin{array}{c}\text { Frreur } \\
\text { relative } \\
\%\end{array}$ & $\begin{array}{c}\text { quantité } \\
\text { calculée } \\
\mu g\end{array}$ & $\begin{array}{c}\text { Erreur } \\
\text { relative } \\
\text { o }\end{array}$ \\
\hline A & $C r=-0,12+82,7$ D. 0 & 10,05 & 0,5 & 19,97 & 0,15 & 30,02 & 0,06 \\
\hline B & $C r=+0,15+80,6$ D. 0 & 9,33 & 6.7 & 21,02 & 5,1 & 29,49 & 1,5 \\
\hline $\mathrm{c}$ & $C r=+0,32+99,9$ D. 0. & 9,1 & 8.9 & 21.1 & 5.5 & 29,5 & 1.5 \\
\hline $\mathrm{D}$ & $C r=-0,91+105 \quad$ D. 0. & 11,1 & 10,6 & 20,9 & 4,6 & 28,9 & 3,3 \\
\hline $\mathrm{E}$ & $C r=-1,11+97 \quad D .0$. & 11,5 & 14,8 & 20,98 & 5 & 28,67 & 4,4 \\
\hline
\end{tabular}




\section{5. - Vérification de la lol de BEER-LAMBERT avec du sérum sanguin}

Au cours de cette vérification, des quantités croissantes de sérum déféqué ont été mises en œuvre, la déprotéination étant conduite de la manière suivante :

$$
\begin{aligned}
& \text { Sérum sanguin............ } 2 \mathrm{ml} \\
& \text { Eau distillée............. } 3 \mathrm{ml} \\
& \text { Na tungstate à } 10 \text { p. } 100 \ldots \ldots .1 \mathrm{ml} \\
& \mathrm{H}_{2} \mathrm{SO}_{4}(0,67 \mathrm{~N}) \ldots \ldots \ldots \ldots 2 \mathrm{ml}
\end{aligned}
$$

On récupère le surnageant obtenu par centrifugation et on le soumet à l'action de $20 \mathrm{cg}$ de Jagolithe $A$, puis à celle du réactif picro-sodé.

Les résultats obtenus sont les suivants :

$\begin{array}{cc}\begin{array}{c}\text { Sérum sanguin } \\ \text { utilisé, } \mathrm{ml}\end{array} & \text { quantité calculée } \\ 1 & \mathrm{ml} \\ 1 & -\overline{0} \\ 1,25 & 1,02 \\ 1,50 & 1,28 \\ 1,75 & 1,50 \\ 2 & 1,72 \\ & 1,98\end{array}$

Par conséquenti, qu'il s'agisse de solutions pures de créatınine ou de sérum sanguin déféqué, il existe une proportionnalité entre la densité optique et les quantités de créatinine mises en jeu.

\section{6. - Essais de récupération de surcharges de créatinine}

Avant défécation, on ajoute au sérum des quantités croissantes de créatinine. Nous reproduisons ići des résultats d'un essai qui montrent une récupération satisfaisante des surcharges ainsi réalisées.

$\begin{array}{cc}\text { Surcharge réalisée } & \text { Surcharge récupérée } \\ \mu g \text { Créatinine } & \mu g \text { Créatinine } \\ - & - \\ 0 & 0 \\ 2,5 & 2,6 \\ 5 & 5,1 \\ 7,5 & 7,4 \\ 10 & 10,2 \\ 15 & 15,1\end{array}$

La déprotéination du sérum ne se traduit donc pas par une perte de Créatinine.

\section{7. - Sélectiviłé de l'adsorption}

Parmi les nombreuses subsiances qui peuvent donner une réaction Jaffe positive, nous avons testé le comportement de l'acétone et du glucose.

En solution, ces substances ne sont pas adsorbées par la Jagolithe. Ajoutées à des solutions pures de créatinine ou aux sérums, elles ne modifient pas les résultats des dosages.

\section{8. - Technique de dosage adoptée pour la créatinine sanguine}

\section{A. - Réactifs :}

1) Solution mère de créatinine :

Créatinine pure Merck ..... $\quad 0,250 \mathrm{~g}$

$\mathrm{HCl} 0.1 \mathrm{~N} \ldots \ldots \ldots \ldots . \mathrm{qs} \quad 250 \mathrm{ml}$

conserver en flacon teinté sous toluène.

2) Solution de créatinine d $10 \mu \mathrm{g} / \mathrm{cm}^{3}$ :

Préparer extemporanément à partir de lá solution mère par dilution au $1 / 100$.

3) Acide picrique recristallisé de l'acide acétique:

Dissoudre $100 \mathrm{~g}$ d'acide picrıque dans $150 \mathrm{ml}$ d'acide acétique glacial dans un Erlenmeyer, en chauffant. Poursuivre le chauffage jusqu'à ébulition. Filtrer sur filtre à pl is dans un entonnoir sec et chaud. Recueillir le filtrat dans un flacon $\mathrm{sec}$. Recouvrir d'un verre de montre ef laisser une nuit à la température ambiante. Filtrer sous vide sur verre fritté, laver avec de l'acide acétique glacial froid. Continuer l'aspiration jus. qu'à disparition dé l'odeur d'acide acétique et sécher à 80-90' en maintenant un courant d'air.

4) Solution solurée d'acide picrique :

Acide picrique recristallisé
Eau distillée $\quad \ldots \ldots \ldots \ldots . \quad 1,2 \mathrm{~g}$

5) Réactif picro-sodé :

Solution saturée d'acide picrique $55 \mathrm{ml}$ $\mathrm{NAOH}(2,5 \mathrm{~N}) \ldots \ldots \ldots$. Qs $11 \mathrm{ml}$ Eau distillée .......... Qs $200 \mathrm{ml}$

6) Acide sulfurique $0,67 \mathrm{~N}$

7) Sodium tungstate, solution aqueuse 10 p. 100 à préparer extemporanément. 
8) Préparotion de la jagolithe A

Placer l'argile pendant une nuit à l'étuve à $100-105^{\circ} \mathrm{C}$. Broyer finement au mixer. Tamiser et conserver la Jagolithe traversant la maille de bronze no 190.

\section{B. - Technique :}

a) défécotion du sérum :

Introduire dans un tube à centrifuger conique :

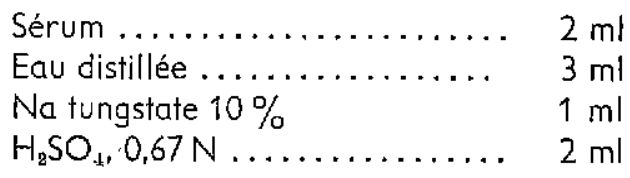

Mélanger avec un agitateur, Centrifuger 10 minutes. Décanter le surnageant.

b) Préparation de lo gamme étolon :

Introduire dans trois tubes à centrifuger coniques:

Solution créatinine à

$10 \mu \mathrm{g} / \mathrm{cm}^{3} \ldots \ldots \ldots \ldots \quad 0 \quad 1 \mathrm{ml} \quad 2 \mathrm{ml}$

Equ distillée, $\mathrm{ml} \ldots \ldots . \quad 5 \quad 4 \quad 3$

Na tungstate 10 p. $100 \quad 1 \quad 1 \quad 1$

$\mathrm{H}_{\mathrm{a}} \mathrm{SO}_{4}, 0,67 \mathrm{~N}, \mathrm{ml}$.

$\begin{array}{ccc}\text { tube } 0 & \text { tube } 1 & \text { tube } 2 \\ - & - & \stackrel{1}{0} \\ 0 & 1 \mathrm{ml} & 2 \mathrm{ml} \\ 5 & 4 & 3 \\ 1 & 1 & 1 \\ 2 & 2 & 2\end{array}$

c) Extraction de la créatinine.

Introduire dans un tube à centrifugè conique $4 \mathrm{ml} \mathrm{du}$ liquide de centrifugation des sérums déprotéinés.

Ajouter dans tous les tubes (sérums déproteinés et gamme étalon), $25 \mathrm{cg}$ de Jagolithe A (une mesure peut être confectionnée à cet effet).

Agiter avec une baguette de verre trois fois en dix minutes. Rincer l'agitateur. Centrifuger pendant 10 minutes. Rejeter le liquide surnageant, laisser égoutter les culots de centrifugation.

d) Développement de la coloration.

Ajouter dans chaque tube $7,5 \mathrm{ml}$ de réactif picro-sodé. Agiter pour remettre le culot en suspension trois fois en dix minutes. Centrifuger pendant dix minutes et recueillir le surnageant dont on détermine la densité optique à $520 \mathrm{~m} \mu$.

e) Calculs :

A l'aide de la gamme étalon, établir la formule de régression donnant la concentration en fonction de la densité optique, (méthode des moindres carrés). Utiliser cette formule pour le calcul des concentrations sanguines.

Laboratoire national de recherches vétérinaires de Dakar-Hann-Sénégal

Laboratoire de biochimie.

\section{RÉSUMÉ}

La créatinine sanguine peut être électivement adsorbée sur une argile montmorillonite, la terre de BEZENET (Jagolithe). Après élution, la créatinine peut être soumise d̀ un dosage colorimétrique basé sur la réaction de JAFFE. La méthode proposée repose sur les points expérimentaux suivants :

10 Le pouvoir adsorbant de l'argile est d'autant plus fort que la taille des grains est plus faible. Il est inférieur à celui du réactif de Lloyd.

$2^{\circ}$ Les spectres d'absorption lumineuse des complexes colorés créatinine-acide picrique dépendent de la quantité et de la granulation de la Jagolithe utilisée. Avec une argile grossière le maximum d'absorption se déplace vers les grandes longueurs d'onde tandis que l'intensité de cette absorption diminue pour les courtes longueurs d'onde.

30 Lo coloration propre du réactif picro-sodé s'intensifie légèrement au contact de l'argile.

40 Les complexes colorés obtenus après élution suivent de très près da loi de BEER-LAMBERT lorsqu'on utilise une argile traversant les tamis no 190 . Avec des granulations plus fortes, les résultats sont moins bons.

$5^{\circ}$ Avec le sérum sanguin déprotéiné ( $\mathrm{Na}$ tungstate- $\mathrm{SO}_{4} \mathrm{H}_{2}$ ) on note également une bonne proportionnalité entre coloration et concentration.

$6^{\circ}$ Les surcharges de créatinine ajoutées au sérum sont quantitativement récupérées. 


\section{SUMMARY \\ Use of montmorilionite (the clay of Benezet) \\ in the selective dosage of the blood oreatinine of the domestic ruminants}

The blood creatinine can be electively adsorbed upon a montmorillonite, the clay of Benezet (Jagolithe). After elution, the creatinine can be estimated by the colorimetric dosage based on the reaction of Jaffe. The proposed method is based on the following experimental points:

10 The adsorbing capacity of the clay is the higher as the size of the grains is small. It is inferior to one of the Lloyd's reagent.

$2^{0}$ The spectro of luminous absorption of the coloured complexes creatinine-picric acid, are dependent on the quantity and the granulation of the Jagolithe used. When a coarse clay is used, the maximum absorption is seen with the long wave-lengthes whereas the intensity of absorption decreases for the short wave-lengthes.

30 The colouration itself of the reagent picro-sodic increases slightly by contact with the clay,

40 The coloured complexes obtained after elution follow very closely the law of Beer-Lambert when the clay filtered through sieves $n^{0} 190$ is utilized. The resulis are not so good when bigger granulations are used.

5o With the proteinless blood serum ( $\mathrm{Na}$ tungstate- $\mathrm{H}_{2} \mathrm{SO}_{4}$ ), there is a good proportionality between colouration and concentration.

' $6^{\circ}$ The added overcharges of creatinine to the serum are quantitatively recovered.

\section{RESUMEN}

Utilización de una montmorillonita, la tierra de Bezenet, en la dosificación selectiva de la creatinina sanguina de los rumiantes domésticos

La creatinina sanguina puede quedar electivamente absorbida por una arcilla móntmorillonítica, la tierra de Benezet (Jagolithe). Previa elución, la creatinina puede quedar sometida a una dosificación colorimétrica fundada en la reacción de Jaffe. El método propuesto toma apoyo en los puntos experimentales siguientes :

10 El poder adsorbente de la arcilla es tanto más fuerte cuanto más reducida es la diménsión de los granos, siendo inferior al del reactivo de Lloyd.

2o Los espectros de absorción luminosa de los complejos coloreados creatinina-ácido pícrica dependen de la cantidad y de la granulación de la Jagolithe utilizada. Con una arcilla basta, la absorciôn mâxima se desplaza hacia las grandęs longitudes de onda, mientras que la intensidad de esta absorción disminuye para las cortas longitudes de onda.

30 La coloración propia del reactivo picro-sodado se intensifica ligeramente al entrar en contacto con la arcilla.

40 Los complejos coloreados obtenidos después de la elución son muy semejantes a las condiciones de la ley de Beer-Lambert cuando se utiliza una arcilla que pasa por la malla 190. Cyando se opera con granulaciones mas fuertes, los resultados son menos buenos.

$5^{\circ}$ Con el suero sanguíneo desproteinado ( $\mathrm{Na}$ Tungstato- $\mathrm{SO}_{4} \mathrm{H}_{2}$ ) se pone de manifiesto, asimismo, una buena proporcionalidad entre coloración y concentración.

$6^{\circ}$ Las sobrecargas de creatınina añadidas al suero quedan quantitatıvamente recuperadas.

\section{BIBLIOGRAPHIE}

KAYSER, (F.), MOLITOR, (A). - Annoles

Pharm. Fronc. (1956) 14, 197.208.
KAYSER, (F.), MOLITOR, (A). - Anח. Biol. Clin., (1956) 14, 224-228.

MEUNIER, (P.), VINET, (A) - Bull. SOC. Chim. Biol., (1943) 25, 327-331. 